\title{
CIÊNCIA'NATURA
}

\section{Comparação entre dois modelos de função footprint aplicados ao sítio experimental de Pedras Altas}

\section{Comparison between two footprint function models applied to the experimental site of Pedras Altas}

\author{
${ }^{1}$ Tiago Bremm, ${ }^{1}$ Ivan Mauricio Cely Toro, ${ }^{1}$ Gustavo Pujol Veeck, ${ }^{1}$ Débora Regina Roberti e \\ ${ }^{1}$ Lidiane Buligon
}

${ }^{1}$ Universidade Federal de Santa Maria, Santa Maria, RS, Brasil

\section{Resumo}

Torres micrometeorológicas são instaladas em diversas regiões a fim de coletar dados atmosféricos em alta frequência para a estimativa de parâmetros micrometeorológicos e de fluxos superficiais de energia e massa. A determinação do campo de visão das torres e, portanto, do fluxo medido nos sensores é definido pelo footprint, o qual é diretamente influenciado pela estrutura da geometria do terreno em que o sensor está instalado e pela vegetação do local. Desta forma, o presente trabalho tem como objetivo analisar a área de contribuição predominante nos fluxos superficiais através de dois diferentes métodos de determinação do footprint que consistem em: um modelo analítico e outro estocástico lagrangiano. Os dados analisados foram da estação micrometeorológica de Pedras Altas, localizada na região sudoeste do Rio Grande do Sul. Os resultados mostram que o modelo estocástico considera o campo de visão da torre mais próximo que o modelo analítico, consequentemente abrange uma área menor.

Palavras-chave: Footprint, micrometeorologia, fuxo superficial.

Abstract

Micrometeorological towers are installed in several regions in order to collect atmospheric data at high frequency for the estimation of micrometeorological parameters and surface flows of energy and mass. The determination of the towers field of view and, therefore, the flow measured at the sensors is defined by the footprint, which is directly influenced by the geometry of the terrain and by the vegetation of the site on which the sensor is installed. In this way, the present work aims to analyze the predominant area of the flow contribution by two different methods of footprint determination: one analytical model and one lagrangian stochastic. The data were analyzed for the micrometeorological station of Pedras Altas, located in the south western region of Rio Grande do Sul. The results show that the stochastic model considers the tower field of view closer than the analytical model, consequently it covers a smaller area.

Keywords: Footprint, micrometeorology, superficial flux. 


\section{Introdução}

Considera-se como local ideal para a instalação de uma torre micrometeorológica ou torre de fluxo, um terreno plano e homogêneo, com cobertura de vegetação distribuída igualmente em toda a superfície. Além disso, o tamanho da área em que o sensor está instalado deveria ser suficientemente maior que a extensão da área que se deseja medir. Contudo, a maioria dos sítios não atende essas hipóteses, fazendo com que informações de rugosidade superficial sejam necessárias para a interpretação da área de contribuição do fluxo (Rannik et al., 2012).

O footprint de uma torre de fluxo pode ser definido como a determinação do campo de visão das torres. Ele deve ser determinado considerando o fluxo não homogêneo, devido à vegetação e terreno irregulares. Porém, grande parte dos modelos empregados na determinação do campo de visão de torres micrometeorológicas são analíticos e, consideram apenas o fluxo homogêneo. Esses modelos, por serem mais simples, não exigem muita capacidade computacional e mesmo assim, resultam em boas estimativas. Para estimativas sobre terrenos mais complexos e superfícies ou vegetação irregulares os modelos estocásticos lagragianos são os mais indicados (Rannik et al., 2012).

Nesta perspectiva este trabalho busca calcular o footprint por meio de dois métodos distintos, método analítico (Kormann e Meixner, 2001) e estocástico lagrangiano (Kljun et al., 2015) que foi adaptado do modelo de Kljun et al. (2004). O método analítico considera o terreno e a vegetação homogêneos, enquanto o modelo lagrangiano estocástico considera terreno complexo ou irregular e vegetação não homogênea.

\section{Materiais e Métodos}

Os dados utilizados neste trabalho foram coletados na estação micrometeorológica ( $31^{\circ} 43.556^{\prime}$ S, 53 $\left.32.036^{\text {' }} \mathrm{W}, 395 \mathrm{~m}\right)$ localizada na cidade de Pedras Altas, sudoeste do Rio Grande do Sul, e caracterizada como bioma pampa. Foram utilizados dados nas direções $u, v$ e $w$ do vento, obtidos pelo Anemômetro Sônico (CSAT3/CampbellSci) instalado a 2,5 m de altura do solo. A altura da vegetação foi estimada em $15 \mathrm{~cm}$.

O período de análise corresponde à média diária das variáveis para o dia 10 de janeiro do ano de 2014.

\subsection{Modelo analítico}

O modelo analítico de footprint proposto por Kormann e Meixner (2001) foi determinado através da versão 1.0 do pacote FREddyPro (Xenakis, 2016), utilizado no software R para computação estatística (R Core Team, 2017).

O problema da difusão estacionária pode ser resolvido introduzindo-se a equação da continuidade, que na aproximação de Kormann e Meixner (2001) à equação de advecção-difusão bidimensional:

$$
u \frac{\partial c}{\partial x}=-\frac{\partial f}{\partial z}
$$

Seguindo estes autores, o fluxo turbulento vertical e assim, o footprint, é descrito por um gradiente de difusão vertical que, integrado na direção $y$ do vento, resulta em:

$$
f(x, z)=-\frac{K}{\bar{u}} \frac{\partial D_{z}}{\partial z}=-K \frac{\partial c(x, z)}{\partial z}
$$

Assumindo que o perfil vertical da velocidade horizontal do vento e a difusividade turblenta são dados, respectivamente, por uma função positiva como segue:

$$
\begin{aligned}
& u(z)=U z^{m} \\
& K(z)=k z^{m}
\end{aligned}
$$

além disso, a concentração integrada do vento lateral por unidade de área, equação (5)

$$
c(x, z)=\frac{A}{\overline{z u}} \exp \left[-\left(\frac{B z}{\bar{z}}\right)^{r}\right]
$$


com A e B constantes, que combinada com a equação (2), resolvem a equação (1).

A equação (5) pode ser reescrita como,

$$
c=\frac{1}{\Gamma(\mu)} \frac{r}{U z^{(1+m)}} \frac{\xi^{\mu}}{x^{\mu}} e^{\frac{-\xi}{x}}
$$

onde $\xi(z)=\frac{U z^{r}}{r^{2} k}$ e $\mu=(1+m) / r$. $m$ e $n$ representam o expoente da lei de potências da velocidade do vento e difusividade turbulenta, respectivamente e, $r=2+m-n$ é o fator de forma.

A equação (2) combinada com as equações (3),(4) e (6) resulta em,

$$
f=\frac{1}{\Gamma(\mu)} \frac{\xi^{\mu}}{x^{1+\mu}} e^{\frac{-\xi}{x}}
$$

que descreve o fluxo integrado do vento à distância $x>0$ e altura $z . \xi$ representa a escala de comprimento do fluxo e $\Gamma$ representa a função gama.

\subsection{Modelo estocástico lagrangiano}

A determinação do footprint através do modelo estocástico lagrangiano foi realizada utilizando a função de Kljun et al. (2015) Segundo estes autores, matematicamente, o footprint é a função de transferência entre fontes ou sumidouros de escalares na superfície e o fluxo turbulento medido no sensor, conforme a equação (8).

$$
f(x, y)=\frac{F_{c}\left(0,0, z_{m}\right)}{Q_{u}(x, y)}
$$

Sendo $F_{c}$ o fluxo de densidade (por unidade de área) e $Q_{u}$ é a fonte ou sumidouro integrado por unidade de área. Assumindo que a dispersão turbulenta do vento pode ser tratada independentemente de transporte vertical ou contínuo, a função do footprint pode ser expressa em termos do footprint integrado do vento $\overline{f^{y}}$ e da função de dispersão, $D_{y}$, conforme ilustrado por Kljun et al. (2015).

$$
f(x, y)=\overline{f^{y}}(x) D_{y}
$$

\section{Resultados}

O footprint foi calculado para o dia 10 de janeiro, classificado como cenário estável segundo Kljun et al. (2015). As medidas micrometeorológicas estão apresentadas na tabela 1. A altura da CLP (h) foi mantida em 300 metros pois, segundo Kljun et al (2015), mesmo uma variação de até $20 \%$ em sua altura causará pouca influência no footprint estimado para cenários estáveis.

Tabela 1: Parâmetros de entrada dos modelos para o dia 10 de janeiro, sendo $u_{*}$ a velocidade de fricção, $L$ o comprimento de Obukhov e $h$ a altura da camada limite.

\begin{tabular}{l|l|r|r} 
Condição & $u_{*}\left(m s^{-} 1\right)$ & $\mathrm{L}(m)$ & $\mathrm{h}(m)$ \\
\hline Estável & 0.294 & 51.89 & 300
\end{tabular}

A figura 1 mostra a pluma do footprint referente ao modelo analítico de Kormann e Meixner (2001). A maior parte da contribuição é proveniente de uma região entre 0 e 50 metros da torre, cerca de $20 \%$ do campo de visão do sensor.

A pluma para o footprint proposta por Kljun et al. (2015) é apresentada na figura 2. A abertura da pluma é menor, o que significa que, segundo este modelo, o campo de visão do sensor é mais próximo da torre que o modelo analítico e, possui um ângulo de visão que também é menor.

A probabilidade do footprint de ambos os modelos é bastante diferente sendo que, no modelo estocástico, cerca de $30 \%$ da probabilidade do footprint é proveniente de uma região que está localizada a uma distância menor que 15 metros da torre.

Na figura 3(a) pode-se verificar essa afirmação e destacar ainda que o modelo analítico possui contribuição acumulada menor próximo da torre. A contribuição acumulada chega a $90 \%$ a 40 metros do sensor para o modelo estocástico. O Modelo analítico apresenta contribuição acumulada menos acentuada, com apenas $50 \%$ de contribuição acumulada a 40 metros da torre. Ambos os modelos convergem para $100 \%$ acima de 100 metros da torre. 


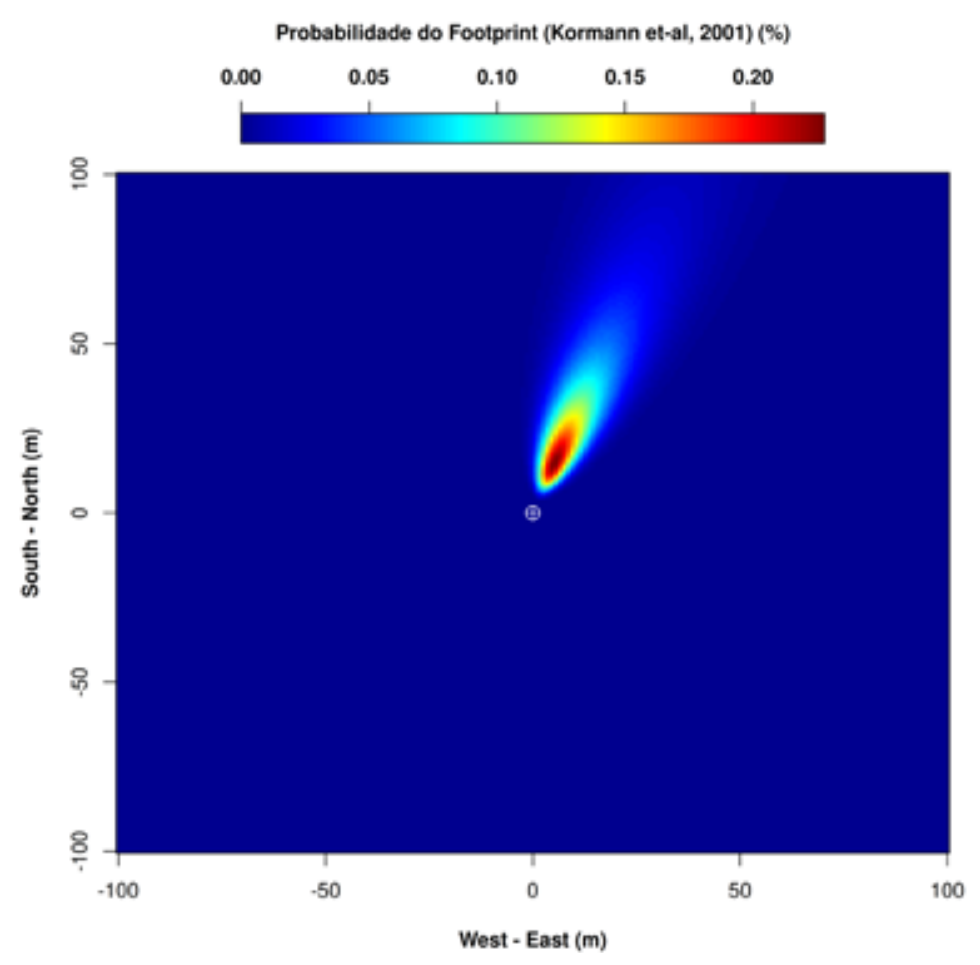

Figura 1: Pluma para o modelo de Kormann e Meixner (2001).

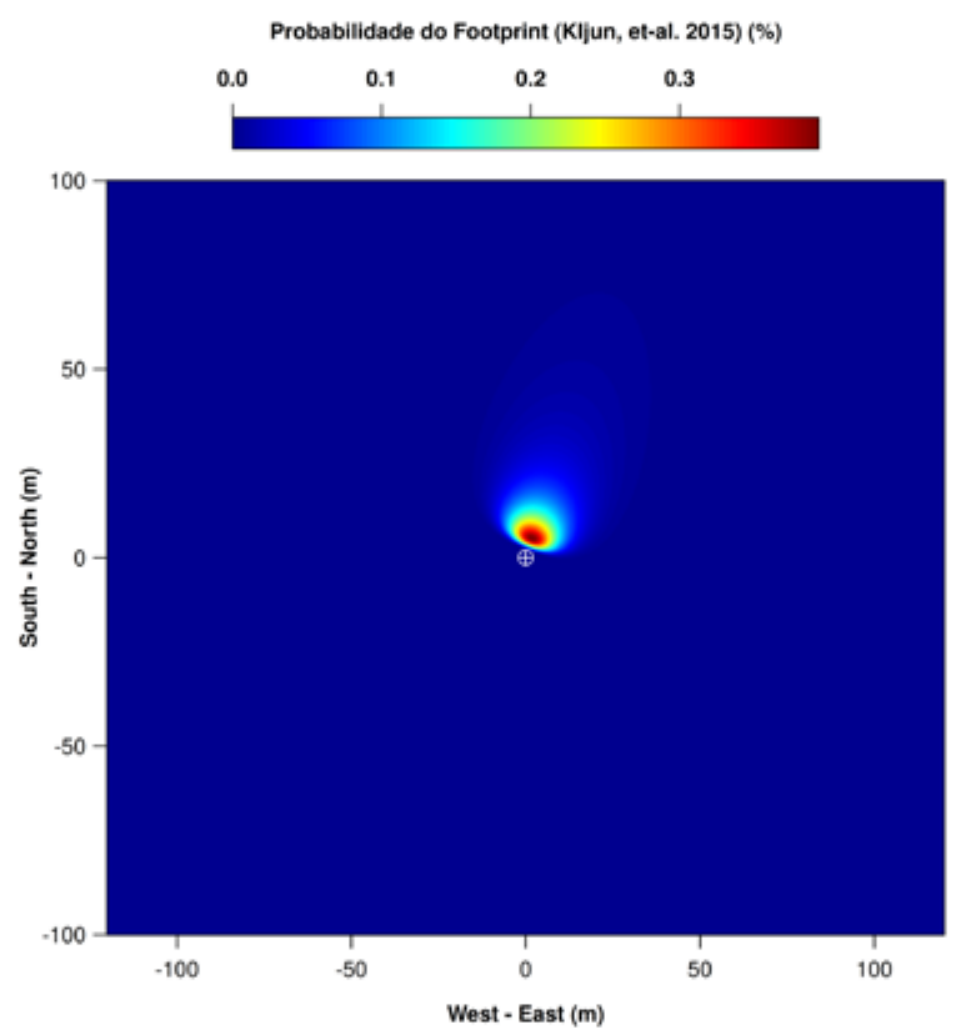

Figura 2: Pluma para o modelo de Kljun et al. (2015). 


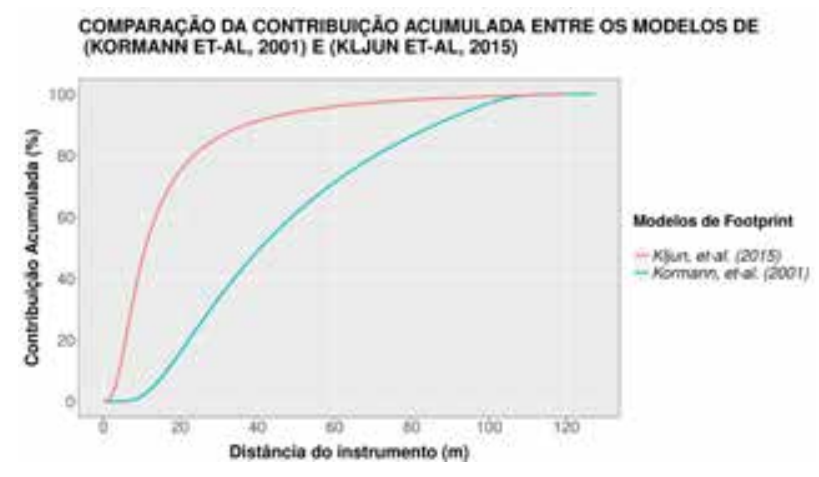

(a) Contribuição cumulativa.

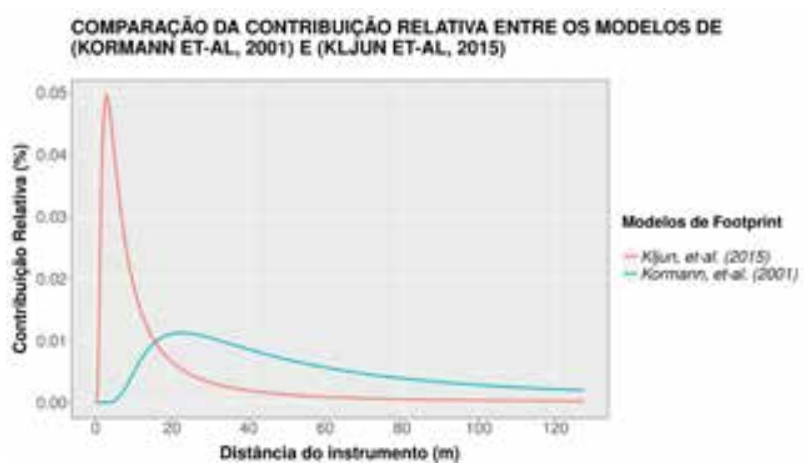

(b) Contribuição relativa.

Figura 3: Contribuições cumulativa e relativa para os dois modelos. A figura 3(a) ilustra a diferença entre a contribuição cumulativa para os modelos analítico e estocástico. A figura 3(b) ilustra a contribuição relativa para ambos os modelos.

A contribuição relativa, apresentada na figura 3(b), mostra que a maior contribuição ocorre a menos de 15 metros da torre, para o modelo estocástico, conforme mostrado também na figura 2. O modelo analítico apresentou maior contribuiação de 20 a 35 metros de distância.

Portanto, é possível verificar que o modelo estocástico apresenta maiores contribuições próximas da torre, consequentemente, melhor utilização para pequenas áreas e para sensores instalados próximos ao solo.

Além disso, observa-se grande diferença na determinação dos footprints estimados para cenários estáveis pelos diferentes modelos. O modelo analítico, que considera a homogeinedade do terreno, apresenta uma área de influência do fluxo turbulento distante da torre, enquanto o modelo estocástico, que considera a não homogeinedade do terreno, apresenta campo de visão menor e próximo da torre.

\section{Conclusões}

O modelo analítico determinou a maior contribuição como sendo entre 20 e 30 metros de distância da torre, o que pode ser explicado pela sua consideração de terreno e vegetação homogêneos. Para áreas muito grandes e sensores muito baixos, esse modelo pode apresentar incoerências na determinação do footprint, devido à necessidade de homogeneidade.

O modelo estocástico apresentou a maior contribuição a menos de 15 metros da torre, o que determina que o campo de visão da torre é menor que o determinado pelo modelo analítico. Essa diferença pode ser explicada pela consideração de terreno não homogêneo e existência de vegetação próximo à torre. Consequentemente ocorre uma limitação no campo de visão da torre, o que diminui a distância de maior contribuição nas medidas de footprint. Este modelo pode apresentar problemas para áreas muito grandes e estimativas incorretas se determinado para cenários convectivos ou altamente convectivos (Kljun et al., 2015).

A partir dessa comparação e considerações, o modelo estocástico é o mais apropriado para áreas pequenas e sensores próximos ao solo. Para outras áreas e condições de estabilidade deverão ser feitas análises para ambos os modelos.

\section{Referências}

Kljun, N., Calanca, P., Rotach, M., Schmid, H. (2004). A simple parameterisation for flux footprint predictions. Boundary-Layer Meteorology, 112(3), 503-523.

Kljun, N., Calanca, P., Rotach, M., Schmid, H. (2015). A simple two-dimensional parameterisation for flux footprint prediction (ffp). Geoscientific Model Development, 8(11), 3695-3713.

Kormann, R., Meixner, F. X. (2001). An analytical footprint model for non-neutral stratification. Boundary-Layer Meteorology, 99(2), 207-224.

R Core Team (2017). R: A Language and Environment for Statistical Computing. R Foundation for Statistical Computing, Vienna, Austria, URL https: //www.R-project.org/.

Rannik, Ü., Sogachev, A., Foken, T., Göckede, M., Kljun, N., Leclerc, M. Y., Vesala, T. (2012). Footprint Analysis, Springer Netherlands, Dordrecht, pp. 211-261. URL https://doi.org/10.1007/978-94-007-2351-1_8. 
Xenakis, G. (2016). FREddyPro: Post-Processing EddyPro Full Output File. URL https: //CRAN.R-project.org/ package=FREddyP ro, r package version 1.0.

\section{Tiago Bremm}

Universidade Federal de Santa Maria, Santa Maria, RS, Brasil E-mail: bremm.tiago@gmail.com

\section{Ivan Mauricio Cely Toro}

Universidade Federal de Santa Maria, Santa Maria, RS, Brasil E-mail: mauriciocelytoro@gmail.com

\section{Gustavo Pujol Veeck}

Universidade Federal de Santa Maria, Santa Maria, RS, Brasil E-mail: veeckgp@gmail.com

\section{Débora Regina Roberti}

Universidade Federal de Santa Maria, Santa Maria, RS, Brasil E-mail: debora@ufsm.br

\section{Lidiane Buligon}

